\title{
La curva de Kuznets en el Departamento del Tolima
}

The Kuznets curve in the Department of Tolima

\section{Autor 1}

William Guillermo Naranjo Acosta

Docente MT, Universidad Nacional Abierta y A Distancia UNAD, CEAD

Ibagué

Correo electrónico: William.naranjo@unad.edu.co

ORCID: https://orcid.org/0000-0002-0223-931X

\section{Autor 2}

Óscar Mauricio Tejada

Docente TC, Universidad Nacional Abierta y A Distancia UNAD, CEAD

Ibagué

Correo electrónico: oscar.tejada@unad.edu.co

ORCID: https://orcid.org/0000-0003-1598-1385

\section{Autor 3}

José Alejandro Vera Calderón

Docente catedrático, Universidad Nacional Abierta y A Distancia UNAD, CEAD Ibagué

Correo electrónico: jose.vera@unad.edu.co

ORCID: https://orcid.org/0000-0003-0752-6446

\section{Resumen}

El presente documento realiza una revisión teórica y empírica de la hipótesis de la curva de Kuznets para el Departamento del Tolima, de acuerdo a la teoría existe una relación empírica entre la desigualdad y el crecimiento económico, en este sentido el siguiente escrito explica la teoría y realiza una contrastación de la hipótesis de Kuznets para el Departamento del Tolima.

En primer lugar, se presenta el marco teórico y el estado del arte del fenómeno en estudio, en segundo lugar, se presenta los datos utilizados, $y$, finalmente, se comprueba la hipótesis en el contexto regional para el Tolima. 


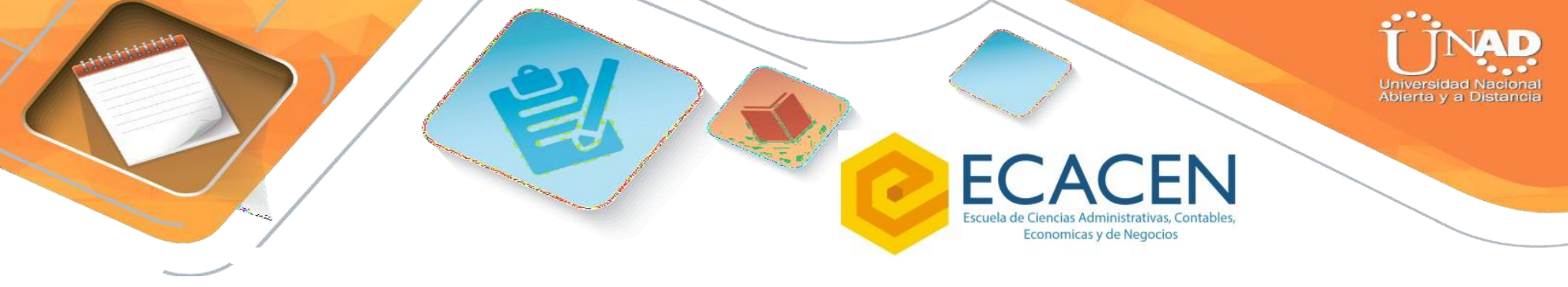

La relación entre la desigualdad y los ingresos presenta una fuerte importancia en los distintos estudios empíricos, ganado cada día más relevancia desde distintos puntos teóricos. A continuación, se presenta un resumen con distintos puntos de vista que permiten comprender la relevancia del tema en estudio.

En primer lugar, es importante relacionar los estudios realizados con la hipótesis de Kaldor, de acuerdo a dicha hipótesis se puede afirmar que, "la propensión marginal a ahorrar de los individuos con mayores ingresos es mayor que la de los individuos pobres" (Fossati, 2002), esto implica que si la variación porcentual del producto interno bruto está correlacionada de manera positiva con la fracción ahorrada del ingreso nacional, las economías más desiguales crecen con mayor rapidez que las economías igualitarias (ingresos), "una economía con mayor desigualdad normalmente tiende a tener más ahorros $\mathrm{y}$, basada en la teoría neoclásica, van a obtener elevados niveles de inversión, así como un incremento en el crecimiento del PIB." (Castillo Lozano, 2019).

Igualmente, la teoría microeconómica y en especial la asimetría de la información, genera acercamientos para explicar los factores que ocasionan el aumento de la producción "el producto depende del esfuerzo no observable de los agentes, recompensarlos con un salario constante, independiente del producto observado, haría que los agentes no inviertan el esfuerzo requerido." (Fossati, 2002), es decir, el nivel de producción ( PIB) está en función de la productividad marginal del trabajo, por tal razón, la ausencia de incentivos ocasiona que los trabajadores se esfuercen menos y con ello disminuya la producción.

Es importante resaltar los estudios realizados por Simon Kuznets en 1995, el autor afirma que el crecimiento económico y la desigualdad tienen una relación en forma de $U$ invertida, esto se conoce como la curva de Kuznets, en el desarrollo de la investigación el autor propone analizar la desigualdad en función del logaritmo natural del producto interno bruto per cápita, adicionando, las variables como, nivel de democracia y gasto público en educación. 
Kuznets finaliza su investigación afirmando "tan pronto como una economía alrededor de su nivel de subsistencia comenzase a desarrollarse, la desigualdad económica aumentaría" (Martínez Navarro, Amate Fortes, \& Guarnido Rueda, 2016), esto se presenta en primer lugar, porque los ahorros se concentran en los individuos de mayores ingresos, es decir, los individuos con mayores ingresos ahorran parte de sus ingresos, mientras los individuos con menores ingresos, el nivel de ahorro es cercano a cero, en segundo lugar, el proceso de industrialización, en la medida que el desarrollo de una economía reemplaza las actividades primarias por las secundarias, ocasionando desplazamiento de individuos del sector rural al sector urbano, generado aumentos en la desigualdad.

Figura 1. Curva de Kuznets

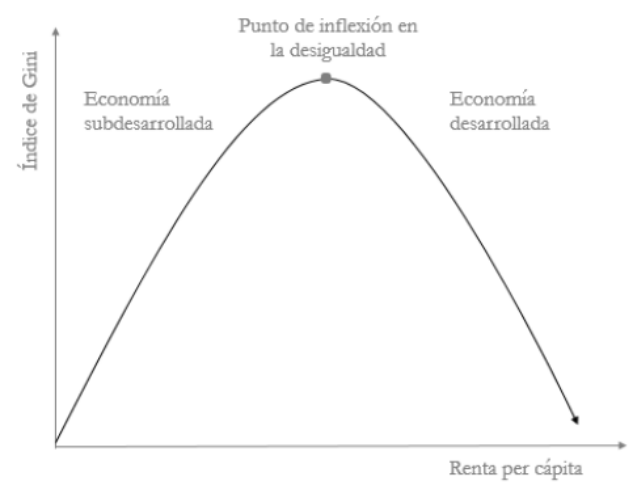

Fuente: (Martínez Navarro, Amate Fortes, \& Guarnido Rueda, 2016)

La Figura anterior resume las ideas de Kuznets "la concentración del ahorro y las migraciones del campo a la ciudad crearían desigualdad en primera instancia" (Martínez Navarro, Amate Fortes, \& Guarnido Rueda, 2016).

De la misma manera se afirma que la "desigualdad como un fenómeno que genera un incremento en la pobreza, sabiendo que las familias se ven 


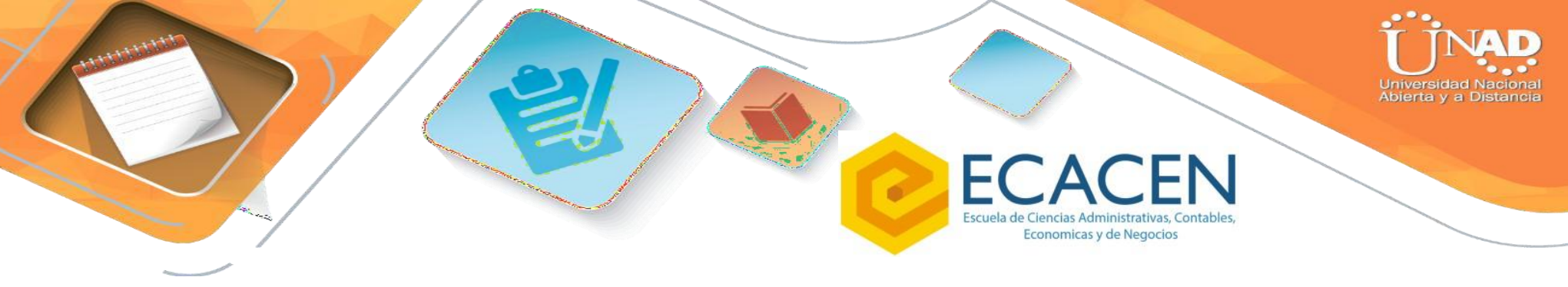

enfrentadas a decidir si tener más hijos y proveer menos educación de calidad o tener menos hijos e invertir más en educación" (Castillo Lozano, 2019), es decir, la desigualdad es un factor que determina la pobreza en una sociedad, llevando a las familias a cambiar sus preferencias de consumo, evidenciando lo poco atractivo que es el gasto en educación en las familias de menos recursos, es decir, el costo de oportunidad entre educación y trabajo es alto, por tal motivo, las familias más pobre ven más rentable la opción de trabajar que estudiar.

Lo anterior expuesto, refleja el aumento de informalidad y un menor de stock de capital humano que respalde o apalanque el crecimiento económico, en la medida que un mejor capital humano afecta de manera positiva la senda de crecimiento económico en los territorios.

Es importante resaltar que "Over the past five years, many economists have attempted to measure this relationship by adding inequality as an independent variable to some variant of Robert J. Barro's cross-country growth regression" (Forbes, 2000) Durante los últimos años el economista Barros mediante el uso de la econometría evidencio que la desigualdad tiene un impacto negativo en el crecimiento económico.

Para ello Forbes presenta el siguiente modelo econométrico, crecimiento económico $=\mathrm{f}$ (desigualdad, los ingresos, educación femenina, educación masculina, nivel de precios de la inversión). Evidenciando que la relación de Baros no es consistente, en la medida que la relación entre desigualdad y crecimiento es positiva y contradiciendo gran parte de la teoría, por tal razón, considera importante una reevaluación teórica a la hipótesis, como también realizar más evidencia empírica adicionando variables omitidas en el debate.

Therefore, this paper suggests the need for not only a further careful reassessment of the reduced-form relationship between these two variables, but also further theoretical and em- pirical work evaluating the channels through which inequality, growth, and any other vari- ables are related. (Forbes, 2000) 
producción del Departamento presentó una tendencia positiva hasta el 2019, el 2020 se evidenció una contracción de la producción, dicha disminución es producto del confinamiento presentado en el país por la llegada del COVID 19, la disminución del 2020 fue de 6,4\% frente al 2019 donde creció $2,4 \%$.

Figura 2. Evolución PIB percapital Tolima

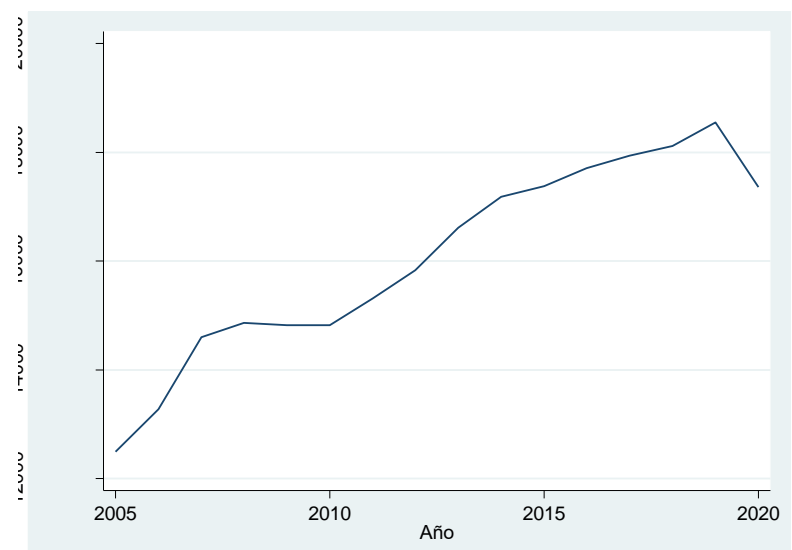

Fuente: Elaboración propia, datos DANE.

Por su parte la desigualdad en el Departamento del Tolima medido desde el índice de GINI, evidencia que en los últimos 14 años estaba descendiendo, pasando de 0,5 en 2006 hasta 0,484 en 2019, para el 2020 producto de la pandemia del COVID 19, la desigualdad en el Tolima presentó un aumento, ubicándose en 0,493.

Figura 3. Evolución de la desigualdad en el Tolima 


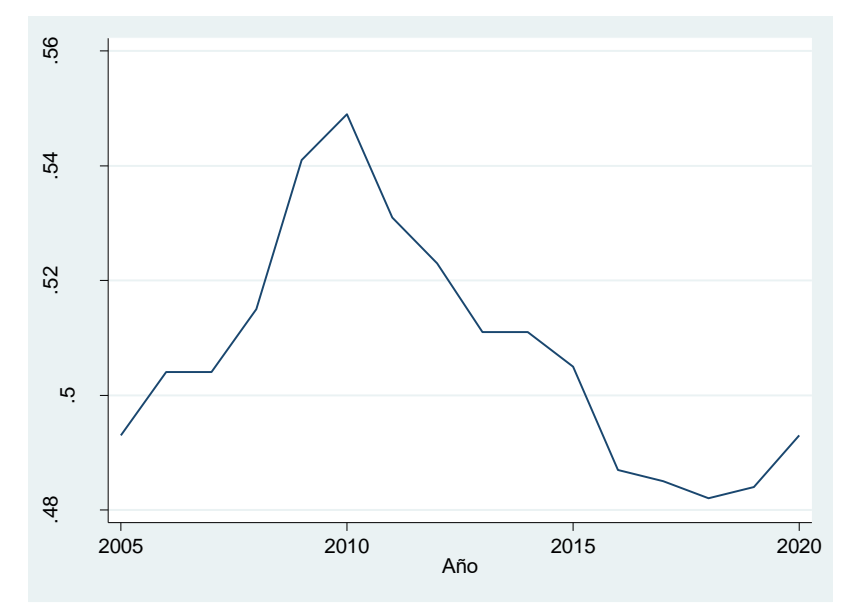

Fuente: Elaboración propia, en base datos DANE

Una vez realizada la base de datos, se aplicó la transformación logarítmica a los datos con el fin de corregir los problemas de ruido blanco que se pueden presentar cuando se trabaja con series de tiempos, luego de la transformación logarítmica se construyó el coeficiente de correlación y la Figura para contrastar la hipótesis de Kuznets en el Departamento del Tolima.

Utilizando el programa Stata14 se realizó la elaboración del coeficiente de correlación entre las variables en logaritmo, identificando que las variables desigualdad y crecimiento económico tiene una dependencia negativa, es decir, el grado de dependencia entre ambas variables es 0,44 , lo que permite concluir que existe una relación inversa entre ambas variables.

Tabla 1. Coeficiente de correlación

\begin{tabular}{r|rr} 
& $\log Y$ & $\operatorname{LogX1}$ \\
\hline $\log Y$ & 1.0000 & \\
$\log X 1$ & -0.4457 & 1.0000
\end{tabular}

Fuente: Elaboración propia en Stata 14. 
La tabla 1 nos indica que mientras aumenta el crecimiento económico se espera que la desigualdad en el Departamento del Tolima disminuya en $44 \%$, lo que nos indica que el coeficiente es consistente con la teoría económica.

Al realizar el gráfico de predicción cuadrática se encuentra que las variables desigualdad y crecimiento económico para el departamento del Tolima, se comporta como el esperado a la curva de Kuznets, el gráfico 4 muestra el comportamiento de las variables en estudio.

Figura 4. Predicción cuadrática

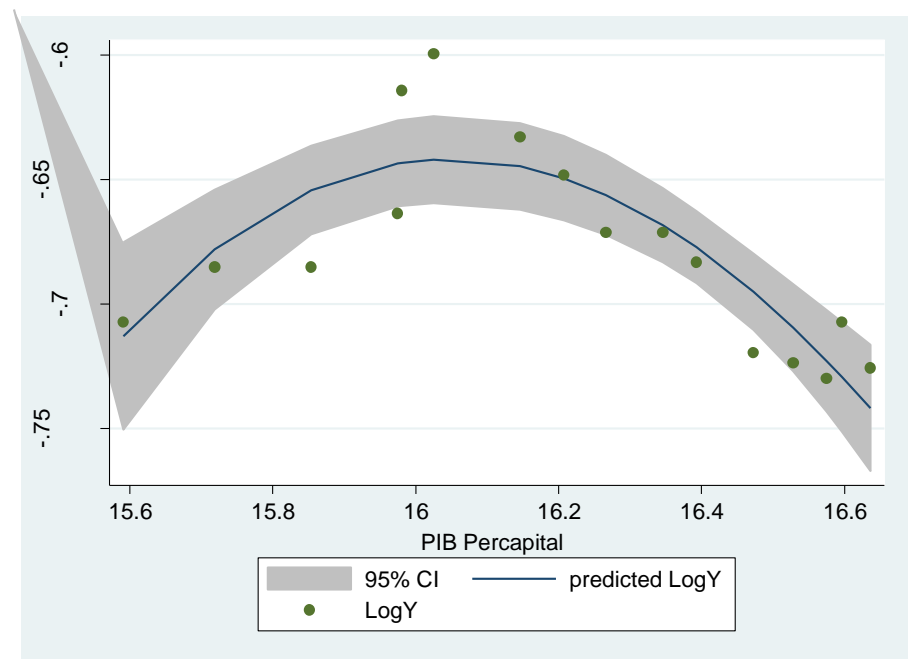

Fuente: Elaboración propia con Stata 14.

Una vez validada la hipótesis de Kuznets, se procedió a correr la regresión propuesta para el modelo en estudio, utilizando el programa Stata se encontró la siguiente salida.

Tabla 2. Salida de la regresión. 


\begin{tabular}{|c|c|c|c|c|c|c|}
\hline Source & SS & $d f$ & MS & \multirow{2}{*}{\multicolumn{2}{|c|}{$\begin{array}{l}\text { Number of obs } \\
\text { F(2, 13) } \\
\text { Prob > F }\end{array}$}} & \multirow{2}{*}{$\begin{array}{r}16 \\
18.43 \\
0.0002\end{array}$} \\
\hline Model & .017724866 & 2 & .008862433 & & & \\
\hline Residual & .006250007 & 13 & .00048077 & $R-s q u$ & tuared & 0.7393 \\
\hline Total & .023974873 & 15 & .001598325 & Root & MSE & .02193 \\
\hline $\log Y$ & Coef. & Std. Err. & t & $P>|t|$ & [95\% Conf & . Interval] \\
\hline $\log X 1$ & 9.828899 & 1.903478 & 5.16 & 0.000 & 5.716684 & 13.94111 \\
\hline $\log X 2$ & -.3059623 & .058922 & -5.19 & 0.000 & -.4332557 & -.178669 \\
\hline _cons & -79.57977 & 15.36827 & -5.18 & 0.000 & -112.7809 & -46.37864 \\
\hline
\end{tabular}

Fuente: elaboración propia con Stata 14.

Al estudiar la bondad de ajuste de la regresión se evidencia, en primer lugar, que los parámetros estimados son estadísticamente significativos a los niveles de significancia de $1 \%, 5 \%$ y $10 \%$, como también, el coeficiente de determinación nos indica que el $73 \%$ de las variaciones en el GINI son explicadas por las variaciones del PIB percapital.

Igualmente, se validó los supuestos de la metodología de mínimos cuadrados ordinarios, encontrando que el modelo realizado cumple con el supuesto de normalidad de los residuos, no presenta multicolinealidad y cumplió la homocedasticidad.

Figura 5. Distribución de los errores de la regresión.

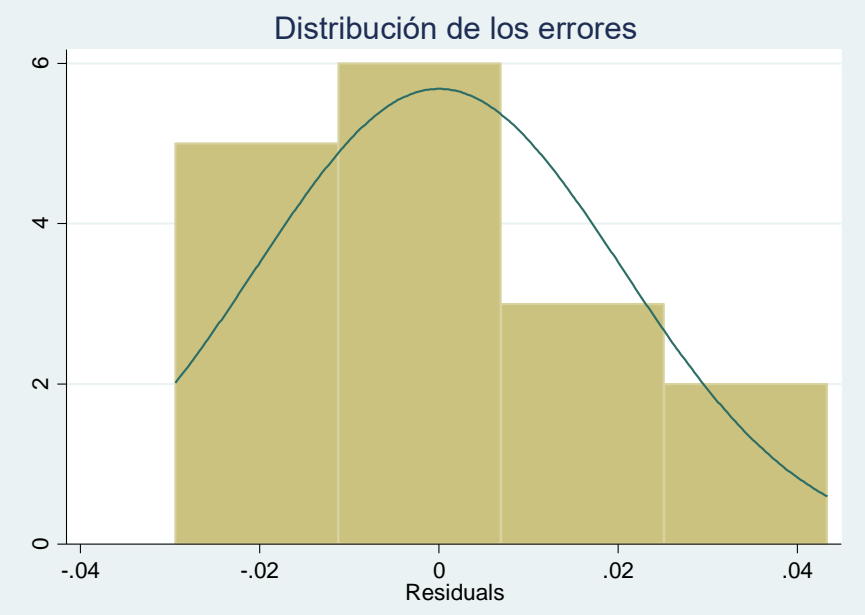




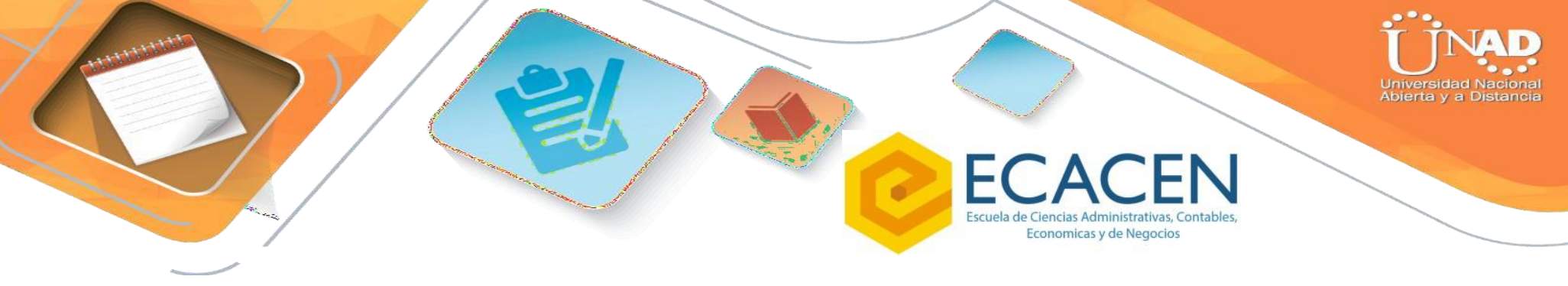

Departamento con el fin de encontrar la propensión marginal al ahorrar y su impacto en el nivel de inversión.

La pandemia del COVID 19 ocasionó un retroceso significativo en la desigualdad en el Departamento del Tolima cercana a 10 años, lo que implica, una mayor intervención del Gobierno con el fin de generar oportunidades de ingresos a los deciles más bajos de la población.

\section{REFERENCIAS}

Castillo Lozano, D. A. (2019). El efecto de la desigualdad sobre el crecimiento económico departamental en Colombia. https://repository.eafit.edu.co/bitstream/handle/10784/15188/Dio nisioAndres_CastilloLozano_2019.pdf?sequence=2\&isAllowed $=y$

Charles, J. A., Mayer, E. L., \& Cheín, N. F. (2018). Desigualdad en el ingreso y crecimiento económico de los municipios mexicanos: el efecto de la natalidad-capital humano. Estudios Económicos, 195217. http://www.scielo.org.mx/pdf/ee/v33n2/0186-7202-ee-3302-195.pdf

DANE. (2020). Departamento Administrativo Nacional de Estadística. https://www.dane.gov.co/index.php/estadisticas-portema/pobreza-y-condiciones-de-vida

Forbes, K. J. (2000). A Reassessment of the Relationship between Inequality and Growth. The American Economic Review, 869-887.

Fossati, V. (2002). Desigualdad y Crecimiento. Un Análisis para las Provincias Argentinas. Buenos Aires: Universidad Nacional de la Plata.

http://sedici.unlp.edu.ar/bitstream/handle/10915/3496/Document o_completo.pdf?sequence $=1$

Martínez Navarro, D., Amate Fortes, I., \& Guarnido Rueda, A. (2016). Desigualdad y desarrollo: ¿Está vigente la curva de Kunzets en la actualidad? Almería: Universidad de Almería. 
Rosales Álvarez,, R. A., \& Bonilla Londoño, J. A. (2006). Introducción a la econometría. Bogotá: Universidad de los Andes. https://economia.uniandes.edu.co/files/profesores/ramon_rosales _alvarez/docs/econometria1/documentos\%20de\%20clase/Introdu ccion_.pdfREVISTA INNOVA ITFIP, 7 (1). 42-56. DIC. 2020 